\title{
Association of Helicobacter Pylori Infection in Patients Having Coronary Artery Disease
}

\author{
A.Ranjit*, C.M. Pathak, K.L.Khanduja, H.K. Ball*, and D.K.Bhasin*
}

Helicobacter pylori usually cause a lifelong infection of gastric mucosa that may lead to gastric ulcer and later on gastric cancer. $\mathrm{H}$, pylori infection and coronary heart disease are common conditions in late middle and old age. Recently, some studies have found casual association between $\mathrm{H}$. Pylori infection and coronary artery disease, cerebrovascular disease and atherosclerosis. The study was done to detect the presence of $\mathrm{H}$. Pylori in patients of coronary artery disease (CAD) by 14C-urea breath test and to find out any possible association between $\mathrm{H}$. Pylori infection and coronary artery disease, The study was conducted in 56 consecutive patients (47M, 9F; Mean age $53.79 \pm 9.2$ yrs ) of coronary artery disease will ECG showing features of myocardial infarction, and 56 spouses or close relatives (29M, 27F: Mean age 39.16 \pm 14.62 yrs ) of the patients having no CAD and staying in the same household served as control. 14C-urea breath test was performed by giving 3 \&\#61549:Ci (111 kBq) of high specific activity, pharmaceutical grade 14C-urea in capsule with water to the overnight lasting subjects and expired 14C02 collected in hyamine hydroxide and the radioactivity measured on LSC. 53.66\% of the myocardial infracted patients were found to be $\mathrm{H}$. Pylori positive as compared to $46.34 \%$ control subjects ( $P>0.05$ ), Among the total population studied, $73.21 \%$-were found to be infected with $\mathrm{H}$. Pylori and out of these $75 \%$ were found to get infected by the age of 55 years. No significant difference was found in the positively between $\mathrm{H}$. Pylori infection and CAD patients when the data was compared with the age and sex matched control subjects taking into account the socio-economic status, the educational level, the source of drinking water, housing conditions, alcoholic or smoking habits; diabetic or hypertensive conditions. Although, there was prevalence of slight increase (\#18776. 7\%) in the incidence of $\mathrm{H}$. Pylori infection in the CAD patients as compared to the control subjects but the results were not statistically significant. Thus, this study does not support any association between $\mathrm{H}$. Pylori infection and coronary artery disease.

Departments of Biophysics, Cardiology' and Gastroentorotogy*, Postgraduate institute of Medical Education and Research, Chandigarh, india 